\title{
The Need for Universal Access to More Flexible Endovascular Stent Graft Constructs
}

Received: September 12, 2016; Accepted: September 15, 2016; Published: September 20, 2016

\section{Editorial}

Abdominal aortic aneurysm (AAA) is the 13th leading cause of death in the United States, responsible for over 15,000 deaths annually. With improved screening methods and the advent of endovascular surgery, mortality from AAA has significantly decreased over the past twenty years [1]. Today it is estimated that $>70 \%$ of aneurysms are amenable to endovascular repair [2]. While endovascular aortic aneurysm repair (EVAR) is confined to the treatment of infra-renal AAA with a $\geq 10 \mathrm{~mm}$ neck, complex endovascular stent grafts have been developed to treat AAA with $<4 \mathrm{~mm}$ infra-renal neck (juxta-renal), involving the renal or visceral vessel orifices (para-renal or para-visceral), or extending to the diaphragm (Type IV thoraco-abdominal aneurysm) or thoracic aorta. It is estimated that $16 \%$ of infrarenal aortic aneurysms are juxta-renal, and a smaller percentage involve the more proximal abdominal aorta [3]. Fenestrated and branched endografts have been developed to treat proximal AAAs not amenable to standard EVAR. However, the extent of what can be treated is limited in the United States by the specific instructions for use (IFU) of each device and what has gone through the rigorous process of FDA approval. Instructions for use outline the recommended anatomic constraints for each device to maximize positive outcomes by guiding patient and device selection. Physicians can treat outside of the IFU but bear significant liability. While certain centers in the US conducting investigational research have access to order endografts outside of their IFU, practitioners in other countries are able to customize devices without these constraints. Patients who could otherwise undergo endovascular repair may be subject to high-risk open surgery, off-label use of devices to perform chimney/snorkel procedures, off-label use of existing FDA approved fenestrated devices, or medical management (at increased risk for aneurysm related death) due to these current restrictions.

Endovascular management of complex aortic aneurysms has been evolving over the past two decades. Browne first described fenestrated endografts in 1999, followed by the application of branched endografts for thoraco-abdominal aneurysm (TAAA) repair by Chuter et al. in 2001 [4,5]. Subsequently, parallel endografts were described by Greenberg et al. in 2003 [6]. Parallel endografts with snorkel, chimney, or encroachment techniques are used to achieve a higher proximal seal while preserving renal flow with a short infra-renal neck. Although

\section{Melissa Baldwin and Rami Tadros}

Division of Vascular Surgery, Department of Surgery, The Icahn School of Medicine at Mount Sinai, New York, USA

\section{Corresponding author: Melissa Baldwin}

\section{Melissa.baldwin@mountsinai.org}

Division of Vascular Surgery, Icahn School of Medicine at Mount Sinai, 1 Gustave L. Levy PI, New York, NY 10029, USA.

Tel: 8132931523

Citation: Baldwin M, Tadros R. The Need for Universal Access to More Flexible Endovascular Stent Graft Constructs. J Vasc Endovasc Surg. 2016, 1:3.

parallel grafts are useful in the acute setting, the long-term outcomes are not established, and there is concern for a higher incidence of proximal type IA endoleak. Branched endografts, currently available off the shelf outside of the United States, are used for complex TAAA where supra-celiac coverage is necessary. The current devices under investigation in the United States are the GORE $^{\circledR}$ EXCLUDER $^{\star}$ Thoracoabdominal Branch Endoprosthesis (TAMBE) and the Zenith t-Branch multi branched stent graft (Cook, Bloomington, Ind). Branched grafts have a main body with four cuffs, which are bridged with stent grafts to the celiac, superior mesenteric, and renal arteries. The mobility of the branch cuffs allows flexible use with a variety of vessel configurations. Fenestrated grafts were initially designed to extend the proximal seal zone above the renal arteries when treating infra-renal aneurysms with short necks $(<10 \mathrm{~mm})$. Most endografts for standard EVAR require a neck $\geq 15 \mathrm{~mm}$, although the Endurant device (Medtronic, Inc., Minneapolis, MN) is approved for $\geq 10 \mathrm{~mm}$ necks. A recent study by Jordan et al. identifying anatomic hostile neck criteria revealed that neck length was an independent predictor of type IA endoleak [7]. This poses the question whether 10-14 mm neck aneurysms may be more appropriately repaired by F-EVAR to decrease the risk of a type IA endoleak. Although off the shelf fenestrated devices are under investigation (Zenith pivot branch device ( $p$-branch) (Cook, Bloomington, Ind)), at the present time fenestrated endografts 
must be custom made over a period of weeks. The most common configuration, used in $66.7 \%$ of patients in the US Multicenter Pivotal Trial, contains two fenestrations for the renal arteries and a scallop for the superior mesenteric artery [8]. The fenestrated main body is paired with a bifurcated component extending into the iliac arteries.

While the majority of patients with AAA will have anatomy amenable to currently available endografts, certain complex aneurysm repairs would only be possible with a device configured outside of its IFU. The Cook Zenith Fenestrated stent graft system (Cook Medical) (ZFEN) was designed for infra-renal aneurysms with a short neck that would not have an adequate seal with a standard EVAR. The ZFEN requires a $\geq 4 \mathrm{~mm}$ infra-renal neck with $<45$ degrees of angulation. The graft may only have three fenestrations or two of each type, including holes or scallops. Additionally, each fenestration has size and positional constraints. For example, the endograft cannot be made when the visceral vessels requiring fenestrations are too far apart and do not all lie within two stent forms. On the other hand, the device cannot be designed for patients with vessels that are too close in clock position, and it is limited in patients with a concomitant aortic dissection. A small diameter or stenotic distal aorta or iliac artery may prevent the use of a bifurcated system, requiring a custom fenestrated aorto-uni-iliac device with subsequent femoral artery bypass.

Beyond the anatomic limitations for F-EVAR in infra-renal aneurysms, certain juxta-renal, para-renal, and thoracoabdominal aneurysms may be amenable to a safer endovascular repair with a unique device configuration. Para-renal and Crawford type IV TAAA would require four vessel fenestrations or branches for repair. A four vessel fenestrated device may allow a shorter region of thoracic aorta to be covered compared to a branched device, lessening the risk of spinal cord ischemia. In certain patients, a mixed configuration stent graft with directional branches and fenestrations may be the best option. Renal fenestrations have a lower rate of occlusion compared to multi-branched stent grafts, whereas a downward branch would best match the anatomy of the SMA [9]. If only one renal artery is within a seal zone for a fenestrated device, a device with a fenestration for the proximal renal artery, a branch for the distal renal artery, and a scallop for the superior mesenteric artery may be ideal. A dominant inferior mesenteric artery would prohibit endovascular surgery, requiring open repair with vessel reimplantation. Since the IMA is often not within two stent forms of the renal arteries, a custom fenestrated device would be necessary. In rare circumstances, accessory renal artery coverage may be essential to achieve a proximal seal during EVAR [10]. It has been performed with evidence of higher renal infarct volume but without adverse clinical effects in patients with normal renal function [11]. However, the use of a custom endograft with fenestrations for the accessory renal arteries may be necessary in a patient with borderline renal function. While less commonly encountered, patients with a prior renal or hepatic transplant may present with an aortic or transplant visceral artery aneurysm requiring F-EVAR with stent graft coverage of the aneurysmal vessel [12].

Complex rescue repair of an endoleak, now requiring para-renal or para-visceral coverage, may also preclude endovascular repair with currently available fenestrated and branched endograft devices [13]. Delivering a device within a short "working" length of a bifurcated graft is technically challenging and may risk dislodgement of the stent graft. This may require placement of a proximal fenestrated cuff without the composite system. The fenestrated cuff would also need a larger distal diameter compared to the proximal portion in order to prevent a type III endoleak. The currently available fenestrated devices may have difficulty passing a high bifurcation leading to unilateral iliac coverage or requiring a modular system with shorter overlapping devices [14,15]. Despite these potential challenges, a high technical success rate using customized stent grafts has been shown in single center studies when performing fenestratedbranched endovascular aortic aneurysm repair (FB-EVAR) after standard EVAR for type la endoleaks, stent migration, or neck degeneration [15]. Cleveland Clinic published their experience with a physician-sponsored investigational device exemption (PSIDE) study where 54 patients underwent FB-EVAR after failed EVAR with an $85 \%$ success rate [16]. In these cases, when a long body graft was in place, they were able to use a fenestrated tube graft with a larger distal diameter to ensure an adequate seal without the need for a bifurcated device. When a short body graft was in place, the design was more complex. The authors used a bifurcated piece with an internal contralateral limb where the fixation is above the flow divider. The high success rate was possible with the ability to design endografts with these unique configurations.

As endovascular surgery evolves, there will be a growing expectation for endovascular management of complex aneurysms. Currently, branched and fenestrated devices must be ordered under strict guidelines, limiting the surgeon's flexibility in management. To maximize positive outcomes with the current technology, physicians who perform complex aortic aneurysm repair in the United States need the ability to design patient specific devices beyond IFU. 


\section{References}

1 Giles KA, Pomposelli F, Hamdan A, Wyers M, Jhaveri A, et al. (2009) Decrease in total aneurysm-related deaths in the era of endovascular aneurysm repair. J Vasc Surg 49: 543-550.

2 Schwarze ML, Shen Y, Hemmerich J, Dale W (2009) Age-related trends in utilization and outcome of open and endovascular repair for abdominal aortic aneurysm in the United States, 2001-2006. J Vasc Surg 50: 722-729.

3 Sarac TP, Clair DG, Hertzer NR, Greenberg RK, Krajewski LP, et al. (2002) Contemporary results of juxtarenal aneurysm repair. J Vasc Surg 36: 1104-1111.

4 Chuter TA, Gordon RL, Reilly LM, Pak LK, Messina LM (2001) Multibranched stent-graft for type III thoracoabdominal aortic aneurysm. J Vasc Interv Radiol 12: 391-392.

5 Browne TF, Hartley D, Purchas S, Rosenberg M, Van Schie G, et al. (1999) A fenestrated covered suprarenal aortic stent. Eur J Vasc Endovasc Surg 18: 445-449.

6 Greenberg RK, Clair D, Srivastava S, Bhandari G, Turc A, et al. (2003) Should patients with challenging anatomy be offered endovascular aneurysm repair? J Vasc Surg 38: 990-996.

7 Jordan WD, Ouriel K, Mehta M, Varnagy D, Moore WM, et al. (2015) Outcome-based anatomic criteria for defining the hostile aortic neck. J Vasc Surg 61: 1383-1390.

8 Greenberg RK, Sternbergh WC, Makaroun M, Ohki T, Chuter T, et al. (2009) Intermediate results of a United States multicenter trial of fenestrated endograft repair for juxtarenal abdominal aortic aneurysms. J Vasc Surg 50: 730-737.
9 Oderich GS, Mendes B (2015) Endovascular Repair of TAAAs with the t-Branch Multibranched Stent Graft. Endovascular Today.

10 Spear R, Maurel B, Sobocinski J, Perini P, Guillou M, et al. (2012) Technical note and results in the management of anatomical variants of renal vascularization during endovascular aneurysm repair. Eur J Vasc Endovasc Surg 43: 398-403.

11 Antoniou GA, Karkos CD, Antoniou SA, Georgiadis GS (2013) Can an accessory renal artery be safely covered during endovascular aortic aneurysm repair? Interact Cardiovasc Thorac Surg 17: 1025-1027.

12 Verzini F, Biello A, Marucchini A, Parente B, Parlani G, et al. (2013) Total endovascular solution for complex visceral aneurysms. J Vasc Surg 58: 1412-1416.

13 Verhoeven EL, Katsargyris A, Oikonomou K, Kouvelos G, Renner H, et al. (2016) Fenestrated Endovascular Aortic Aneurysm Repair as a First Line Treatment Option to Treat Short Necked, Juxtarenal, and Suprarenal Aneurysms. Eur J Vasc Endovasc Surg 51: 775-781.

14 Oikonomou K, Botos B, Bracale UM, Verhoeven EL (2012) Proximal type I endoleak after previous EVAR with Palmaz stents crossing the renal arteries: treatment using a fenestrated cuff. J Endovasc Ther 19: 672-676.

15 Vourliotakis G, Bos WT, Beck AW, Van Den Dungen JJ, Prins TR, et al. (2010) Fenestrated stent-grafting after previous endovascular abdominal aortic aneurysm repair. J Cardiovasc Surg 51: 383-389.

16 Martin Z, Greenberg RK, Mastracci TM, Eagleton MJ, O'Callaghan A, et al. (2014) Late rescue of proximal endograft failure using fenestrated and branched devices. J Vasc Surg 59: 1479-1487. 